\title{
Inference of the optimal probability distribution model for geotechnical parameters by using Weibull and NID distributions
}

\author{
Fengqiang Gong ${ }^{1}$, Tiancheng Wang ${ }^{2}$, Shanyong Wang ${ }^{3}$ \\ ${ }^{1,2}$ School of Resources and Safety Engineering, Central South University, \\ Changsha, Hunan, 410083, P. R. China \\ ${ }^{3}$ ARC Centre of Excellence for Geotechnical Science and Engineering, Faculty of Engineering and Built \\ Environment, The University of Newcastle, Callaghan, New South Wales, 2308, Australia \\ ${ }^{1}$ Corresponding author \\ E-mail: 1'fengqiangg@126.com, ${ }^{2}$ max-tcwang-2016@csu.edu.cn, ${ }^{3}$ Shanyong.Wang@newcastle.edu.au
}

Received 21 February 2018; received in revised form 26 April 2018; accepted 9 May 2018 DOI https://doi.org/10.21595/jve.2018.19758

Check for updates

Copyright $(\mathrm{C} 2019$ Fengqiang Gong, et al. This is an open access article distributed under the Creative Commons Attribution License, which permits unrestricted use, distribution, and reproduction in any medium, provided the original work is properly cited.

\begin{abstract}
To obtain the optimal probability distribution models of geotechnical parameters, the goodness of fit of the normal information diffusion (NID) distribution and Weibull distribution were investigated and compared for actual engineering samples and Monte Carlo (MC) simulated samples. Two datasets from actual engineering parameters (the strength of a rock mass and the average wind speed) were used to test the fitting abilities of these two distributions. The results show that the parameters of the NID distribution are easily estimated, the Kolmogorov-Smirnov $(\mathrm{K}-\mathrm{S})$ test results of the NID distribution are smaller than those of the Weibull distribution, and the NID distribution curves can perfectly reflect the stochastic volatility of geotechnical parameters with small sample sizes. The sample size effects on the fitting accuracy of the NID distribution and Weibull distribution were also investigated in this paper. Eight simulated samples with different sample sizes, namely, 15, 20, 30, 50, 100, 200, 500, and 1000, were produced by using the MC method based on two known Weibull distributions. The results show that with an increase in the sample size, the K-S test results of the NID distribution gradually decrease and tend to converge, while the chi-square test results of the NID distribution remain low and are always lower than those of the Weibull distribution. The cumulative probability values of the NID distribution are larger than those of the Weibull distribution and are always equal to 1.0000 . Finally, the comparison of the fitting accuracy between the NID distribution and normalized Weibull distribution was also analyzed.
\end{abstract}

Keywords: reliability analysis, geotechnical parameters, the optimal probability distribution, probability density function (PDF), normal information diffusion, Weibull distribution.

\section{Introduction}

Due to the natural attributes of rock materials, the complexity of the geological environment and the randomness of external loading (such as impact loads, seismic response, vibratory action, etc.), uncertainty is inevitable in geotechnical engineering [1-3]. To quantitatively evaluate the influence of this uncertainty, reliability analysis has been widely used in many fields of geotechnical engineering [4, 5], such as slope reliability [6-12], tunnel and underground cavity reliability $[13,14]$, etc. In the reliability analysis of geotechnical engineering under quasi-static loads or vibrations loads, the inference of optimal probability density function (PDF) or cumulative distribution function (CDF) of a geotechnical parameter is one of the most essential tasks; this is the first step in a reliability analysis and plays a central role in ensuring the precision and accuracy of the geotechnical reliability analysis $[15,16]$. Through the comparison and selection of the classical distributions (normal distribution, log-normal distribution, Weibull distribution, gamma distribution, etc.), some previous studies have shown that many geotechnical parameters will accept a Weibull distribution as the optimal PDFs [17-20]. However, there are 
some unsolved issues in the application process of the Weibull distribution. The specific PDF forms of the Weibull distribution are not uniform (including the two-parameter, three-parameter and mixed Weibull distributions), and the parameters of the Weibull distribution, such as the shape parameter $m$, the scale parameter $\sigma$ or the position parameter $\mu$, are sometimes difficult to estimate. In addition, the total cumulative probability value of the Weibull distribution is generally less than 1.0000 because its defined interval does not match the actual finite interval of geotechnical parameters. It is necessary to study the inference method, which more accurately represents the actual distribution.

In recent years, the normal information diffusion (NID) theory has been the focus of the attention of many scholars and has been further developed by C. F. Huang et al. [21, 22]. NID theory provides a new way to study function approximation based on the information assignment method of a fuzzy set. In NID theory, the original information is directly transferred to the fuzzy relation in a way that avoids calculation of the membership function and preserves the original information contained in the original data as much as possible. Due to the advantages of the information diffusion principle, NID theory has been successfully applied to some fields of study, especially to natural disaster and risk assessment [23-25].

In this paper, NID theory was introduced to fit the optimal PDFs or CDFs of geotechnical parameters. Two geotechnical parameters, the strength of a rock mass affected by acid [26] and the average wind speed [27], were used as examples to investigate the goodness of fit in a comparative analysis of the NID distribution and Weibull distribution. In addition, the effect of the sample size on the fitting accuracy of these two distributions was also illustrated with MC simulation samples. The results show that NID distribution can make full use of the sample information to deduce the PDFs of the geotechnical parameters and that its fit is more accurate than that of the Weibull distribution.

\section{Weibull distribution}

In mathematical statistics, the Weibull distribution has a range of function forms, including the two-parameter, three-parameter and mixed Weibull distributions, which are widely used in various fields of research. The specific Weibull distribution function is determined by the shape parameter $m$, the scale parameter $\sigma$ and the position parameter $\mu$. Among these parameters, the most important parameter is the shape parameter, which determines the basic shape of the PDF curve. In addition, the scale parameter effects the scaling of the PDF curve. In the geotechnical engineering reliability, the two-parameter Weibull distribution is one of the most commonly used models $[18,19]$. The PDF of the two-parameter Weibull distribution can be written as Eq. (1):

$F(x ; m, \sigma)=1-\exp \left[-\left(\frac{x}{\sigma}\right)^{m}\right], \quad 0 \leq x, \quad 0<m, \quad 0<\sigma$,

where $F(\cdot)$ denotes the cumulative distribution function. $m$ and $\sigma$ is the shape parameter and the scale parameter, respectively.

\section{NID distribution}

The basic principle of the NID distribution was developed by C. F. Huang [21], and a brief introduction is as follows.

Suppose that the PDF of a random variable $x$ is $f(x)$; then, $\mu(x)$ is defined as a Borel measurable function in $(-\infty,+\infty)$. The diffusion estimation of $f(x)$ can be expressed as Eq. (2):

$f(x)=\frac{1}{n \Delta_{n}} \sum_{i=1}^{n} \mu\left(\frac{x-x_{i}}{\Delta_{n}}\right)$ 
where $\Delta_{n}>0$ is defined as the window width and $\mu(x)$ is defined as a diffusion function $f(x)$. According to the information diffusion process, $\mu(x)$ can be written as Eq. (3):

$\mu(x)=\frac{1}{\sqrt{2 \pi} \sigma} \exp \left(-\frac{x^{2}}{2 \sigma^{2}}\right)$

Substituting Eq. (3) into Eq. (2), the normal information diffusion function can be written as follows Eq. (4):

$f(x)=\frac{1}{\sqrt{2 \pi} n h} \sum_{i=1}^{n}\left\{\exp \left[-\frac{\left(x-x_{i}\right)^{2}}{2 h^{2}}\right]\right\}$

where $h$ denotes the window width of the standard normal diffusion function $\mu(x), n$ denotes the sample size of a random variable, $x_{i}(i=1,2,3, \ldots)$ is the observed values of the random variable, and $x_{\max }$ and $x_{\min }$ are the maximum value and minimum value of $x_{i}$, respectively. According to the principle of choosing the nearest normal information diffusion, $h=\gamma\left(x_{\max }-x_{\min }\right) /(n-1)$, in which $\gamma$ is related to the sample size (Table 4). When the sample size is greater than or equal to $17, \gamma$ is always equal to 1.420693101 . The details of the information diffusion process are discussed in Huang's study [22].

\section{Fitting comparison of the NID distribution and Weibull distribution}

\subsection{Data of actual samples}

In this paper, two datasets from actual engineering parameters (the strength of a rock mass and the average wind speed) were used as the examples, which accepted the Weibull distribution as the optimal PDF in previous studies [26, 27]. The specific data are given in Tables 1 and 2.

Table 1. Sample 1\# data

\begin{tabular}{|c|c|c|c|c|c|c|c|c|c|}
\hline 92 & 107 & 113 & 114 & 119 & 120 & 122 & 127 & 128 & 130 \\
\hline 134 & 141 & 142 & 146 & 147 & 148 & 153 & 156 & 167 & \\
\hline
\end{tabular}

Table 2. Sample 2\# data

\begin{tabular}{|l|c|c|c|c|c|c|c|c|c|}
\hline 4.6 & 5.0 & 5.3 & 5.5 & 5.6 & 5.6 & 5.7 & 5.7 & 6.0 & 6.0 \\
\hline 6.3 & 6.4 & 6.5 & 6.5 & 6.6 & 7.0 & 7.1 & 7.6 & 7.8 & 7.8 \\
\hline 7.9 & 8.1 & 8.2 & 8.9 & 8.9 & 9.0 & 9.0 & 9.7 & 9.9 & 10.2 \\
\hline
\end{tabular}

\subsection{Distribution interval determination for the actual samples}

Normally, the actual distribution interval of geotechnical parameters is limited. The sample values of the geotechnical parameters are no less than zero and cannot approach positive infinity; truncated processing is necessary to determine the distribution interval of geotechnical parameters. Here, we provide a new integral interval standard combining a $3 \sigma$ statistical principle and the effect of skewness $c$ : the value of the left end of the interval should not be less than zero. When $c>0,[\mu-3 \sigma, \mu+(3+c) \sigma]$, and when $c<0,[\mu-(3-c) \sigma, \mu+3 \sigma]$, where $\mu$ and $\sigma$ are the mean and standard deviation of the sample parameter, respectively. The truncated intervals for the two actual samples are given in Table 3 .

\subsection{Distribution parameters of the actual samples}

The parameters of the NID distribution and Weibull distribution are given in Tables 4 and 5 . 
The window width $h s$ of the NID distribution for the samples $1 \#$ and $2 \#$ are 5.9196 and 0.2743 , respectively. The distribution parameters of sample 1\# are obtained from [26] and 1\# belongs to the two-parameter Weibull distribution because its position parameter $\mu$ is equal to zero. For determining the distribution parameters of sample 2\#, compared with the fitting goodness of the three-parameter Weibull distribution obtained from [27] and two-parameter Weibull distribution obtained from the maximum likelihood estimation (MLE) method, as shown in Fig. 1(b), it was found that the two-parameter Weibull distribution can be accepted as the probability distribution more accurately than the three-parameter Weibull distribution.

Table 3. The interval values of the actual samples

\begin{tabular}{|c|c|c|c|c|c|c|}
\hline \multirow{2}{*}{ Sample } & \multirow{2}{*}{ Size } & \multirow{2}{*}{ Mean } & \multirow{2}{*}{ Standard deviation } & \multirow{2}{*}{ Skewness } & \multicolumn{2}{|c|}{ Truncated interval } \\
\cline { 6 - 7 } & & & & & Left & Right \\
\hline $1 \#$ & 19 & 131.8947 & 18.9616 & -0.1464 & 72.2338 & 188.7797 \\
\hline $2 \#$ & 30 & 7.1467 & 1.5684 & 0.3385 & 2.4415 & 12.3827 \\
\hline
\end{tabular}

Table 4. The parameters of the NID distributions

\begin{tabular}{|c|c|c|c|c|c|}
\hline Sample & $n$ & $x_{\max }$ & $x_{\min }$ & $\gamma$ & $h$ \\
\hline $1 \#$ & 19 & 167 & 92 & 1.420693101 & 5.9196 \\
\hline $2 \#$ & 30 & 10.2 & 4.6 & 1.420693101 & 0.2743 \\
\hline
\end{tabular}

Table 5. The results of the K-S test values and CDF values

\begin{tabular}{|c|c|c|c|c|c|c|c|c|}
\hline \multirow{2}{*}{ Sample } & \multicolumn{3}{|c|}{ distribution parameters } & \multicolumn{2}{c|}{ Comparison of the K-S test results } & \multicolumn{2}{c|}{ CDF values } \\
\cline { 2 - 9 } & $m$ & $\sigma$ & $\mu$ & Weibull & NID & $D_{n, 0.05}$ & Weibull & NID \\
\hline 1\# & 7.2500 & 140.3000 & 0.0000 & 0.0969 & 0.0683 & 0.3100 & 0.9917 & 1.0000 \\
\hline 2\# (2P) & 5.0339 & 7.7777 & 0.0000 & 0.1495 & 0.0576 & 0.2420 & 0.9966 & 1.0000 \\
\hline 2\# (3P) & 2.1754 & 3.4344 & 3.4395 & 0.2141 & 0.0576 & 0.2420 & 0.9996 & 1.0000 \\
\hline
\end{tabular}

Note: 2P and 3P denote the two-parameter and three-parameter Weibull distributions, respectively

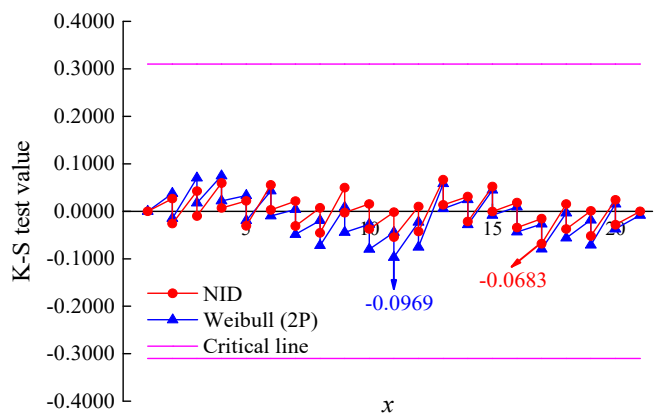

a) Sample $1 \#$

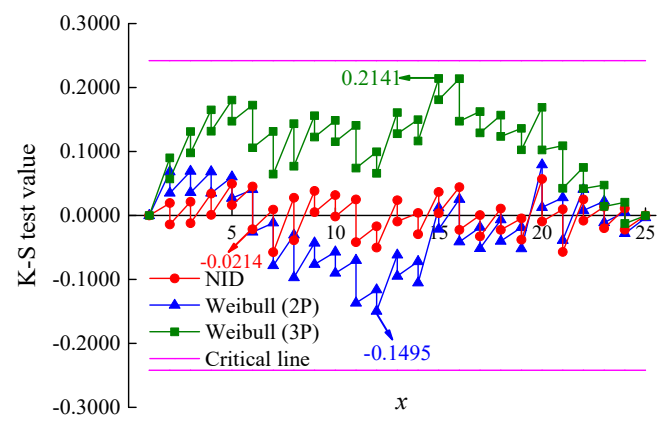

b) Sample 2\#

Fig. 1. Comparative K-S test results of the actual sample data

\subsection{Comparison of goodness of fit}

The K-S test is one of the most widely used goodness-of-fit tests [28]. In this paper, the K-S test was used to discriminate the relative superiority of the NID distribution and Weibull distribution, and the differences between the empirical cumulative frequencies versus theoretical CDF values at every sample point are shown in Fig. 1. The maximum discrepancy of the K-S test results $D_{n} s$, critical values and cumulative probability values of the NID distribution and Weibull distribution are given in Table 5. The critical values of samples $1 \#$ and $2 \#$ are 0.3100 and 0.2420 under $95 \%$ confidence level, respectively. The $D_{n} s$ results of the NID distribution are 0.0683 and 0.0576 , and those of the Weibull distribution are 0.0969 and 0.1495 , respectively. Clearly, both of the Weibull-type distributions pass the K-S testing, while the $D_{n} s$ of the NID distribution are much less than those of the Weibull distribution. In particular, the $D_{n}$ of the Weibull distribution 
is 2.6 times that of the NID distribution for sample 2\#. In addition, both the cumulative probability values of the NID distribution are 1.0000. However, the cumulative probability values of the Weibull distribution are 0.9917 and 0.9966 , respectively. It can be concluded that the fitting accuracy of the NID distribution is higher than that of the Weibull distribution.

\subsection{Comparison of the fitting probability distribution curves}

The empirical cumulative frequency curves and theoretical CDF curves for the two actual sample datasets are given Fig. 2. Within the truncated interval, the goodness of fit of the NID distribution is much more accurate than that of the Weibull distribution.

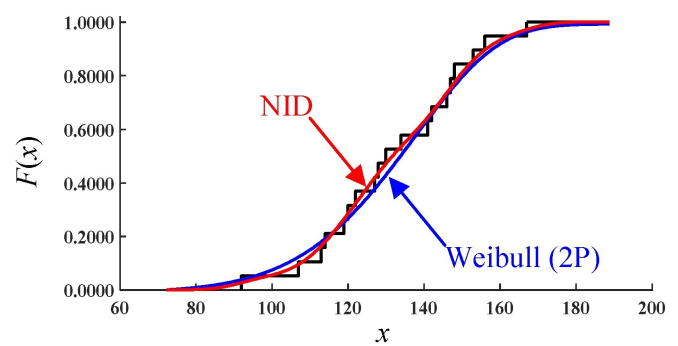

a) Sample $1 \#$

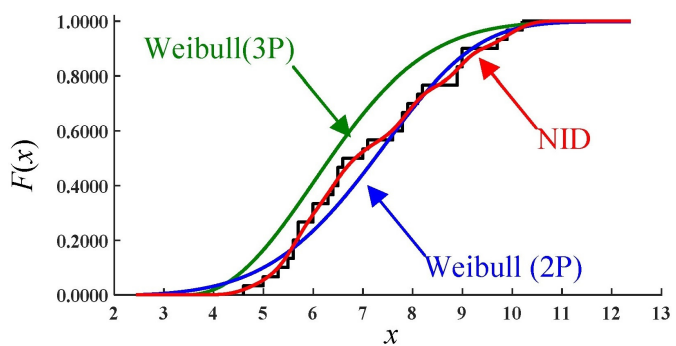

b) Sample $2 \#$

Fig. 2. Comparative CDF curves of the actual sample datasets

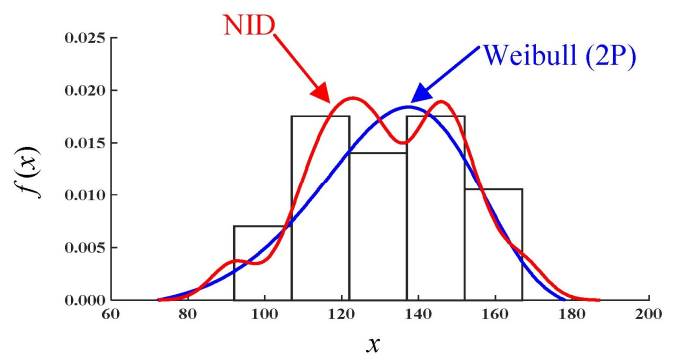

a) Sample $1 \#$

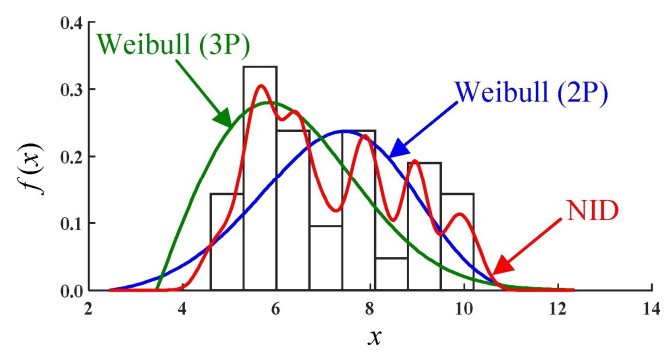

b) Sample 2\#

Fig. 3. Comparative PDF curves of the actual sample datasets

The PDF curves and histograms for the two actual samples are also given in Fig. 3. Due to the uncertainty in and complexity of the geotechnical parameters, the distributions of the actual samples often present a certain fluctuation. As one of the single-peak distributions, the Weibull distribution cannot be used to describe the characteristics of the fluctuation in the actual distribution. However, the NID distribution is very flexible and can be used to describe this fluctuation (Fig. 3).

To summarize, whether for CDF curves or PDF curves, the NID distribution will approximate the actual distribution more accurately than the Weibull distribution will. The superiority of the NID distribution can be further confirmed by describing the actual distributions of the geotechnical parameters.

\section{Effect of sample size on fitting accuracy}

Considering that the sample sizes obtained in actual geotechnical engineering are generally small, to study the effect of the sample size on the fitting accuracy with the NID distribution and Weibull distribution, eight simulated samples of different sizes were produced by using the MC method in this paper. Two known Weibull distributions estimated by samples 1\# and 2\#, WBL1\# $(7.2500,140.3000)$ and WBL2\# $(5.0339,7.7777)$, were used as the generating functions in the MC method, and the simulated sample sizes are 15, 20, 30, 50, 100, 200, 500, and 1000 (partial 
sample datasets are shown in Table 6).

The K-S test was first used to test the validity of the NID distribution and Weibull distribution. The K-S test results and critical values under different sizes are given in Table 7 and Table 8 . The effect of the sample size on the K-S test results is shown in Fig. 4. With an increase in the sample size, the K-S test results of the two fitting methods gradually decrease and tend to converge to the horizontal axis. However, compared with the K-S test results of the Weibull distribution, those of the NID distribution are much lower. The convergence speed and stability and the K-S test results of the NID distribution are all superior to those of the Weibull distribution.

In addition, the chi-square test was used to investigate the fitting ability for all the samples with a sample size larger than 50 . The chi-square test results for a $95 \%$ confidence level are shown in Table 9.

Table 6. Partial simulated samples with the MC method

\begin{tabular}{|c|c|c|}
\hline Size & & Simulated data \\
\hline \multirow{2}{*}{15} & 1\# & $\begin{array}{c}122.1827,111.8577,111.4772,144.3043,143.4258,132.1535,142.5631,111.0938, \\
113.1547,130.3050,146.0154,122.9854,147.7328,135.6769,139.4246\end{array}$ \\
\hline & $2 \#$ & $\begin{array}{c}5.6766,4.9123,8.9816,4.8271,6.6610,9.1989,8.1665,7.0353,4.1709,4.0127,8.7865, \\
3.8716,4.1776,7.2920,5.7718\end{array}$ \\
\hline \multirow[t]{2}{*}{20} & $1 \#$ & $\begin{array}{c}131.3119,72.4864,117.7505,81.6449,147.6651,131.8557,163.0097,117.6235, \\
127.7709,108.4023,76.0449,97.8086,138.1223,186.8482,131.1880,149.3262, \\
148.6061,142.5358,157.7919,118.3333\end{array}$ \\
\hline & 2\# & $\begin{array}{c}9.1276,4.0796,10.8625,5.9287,5.6591,5.2686,9.3094,7.6447,8.2525,5.7731,7.5141, \\
4.8584,8.6470,8.2342,8.8605,8.9211,5.2635,6.8948,7.0227,8.8642\end{array}$ \\
\hline \multirow[t]{2}{*}{30} & $1 \#$ & $\begin{array}{c}118.2600,131.0561,141.8753,111.0446,130.5486,91.0131,103.9049,140.8998, \\
130.8867,141.4201,126.5545,114.3751,118.4575,155.1633,112.0200,167.9393, \\
137.8663,119.5188,115.6696,140.3312,118.5326,103.9849,147.2000,154.8328, \\
148.2517,141.2433,144.6531,98.2004,163.0277,128.3052 \\
\end{array}$ \\
\hline & 2\# & $\begin{array}{c}5.3974,6.7077,7.8491,7.1765,7.6363,9.3872,8.3475,9.0068,8.6356,8.3473,7.5724, \\
9.6763,4.9454,4.3994,7.2694,7.2760,7.9056,4.9734,7.7720,9.0935,5.8966,7.6864, \\
8.3389,7.6276,9.2076,8.9481,4.4431,4.1979,6.9143,9.5544\end{array}$ \\
\hline$\vdots$ & $\vdots$ & $\vdots$ \\
\hline
\end{tabular}

Table 7. The K-S test results and CDF values of sample 1\#

\begin{tabular}{|c|c|c|c|c|c|c|c|}
\hline \multirow{2}{*}{ Size } & \multicolumn{2}{|c|}{ Truncated interval } & \multirow{2}{*}{$D_{n, 0.05}$} & \multicolumn{2}{c|}{ K-S test results } & \multicolumn{2}{c|}{ CDF values } \\
\cline { 2 - 5 } \cline { 6 - 8 } & Left & Right & & Weibull & NID & Weibull & NID \\
\hline 15 & 85.4387 & 171.6572 & 0.3380 & 0.2607 & 0.1113 & 0.9596 & 1.0000 \\
\hline 20 & 31.5233 & 216.2854 & 0.2940 & 0.1476 & 0.0799 & 1.0000 & 1.0000 \\
\hline 30 & 71.3670 & 187.9536 & 0.2420 & 0.1388 & 0.0334 & 0.9923 & 1.0000 \\
\hline 50 & 38.9533 & 196.7983 & 0.1923 & 0.1127 & 0.0200 & 0.9999 & 1.0000 \\
\hline 100 & 46.1519 & 195.3722 & 0.1360 & 0.0894 & 0.0100 & 0.9997 & 1.0000 \\
\hline 200 & 64.3473 & 193.7082 & 0.0962 & 0.0417 & 0.0050 & 0.9965 & 1.0000 \\
\hline 500 & 59.6480 & 196.2190 & 0.0608 & 0.0487 & 0.0020 & 0.9980 & 1.0000 \\
\hline 1000 & 56.5405 & 196.9676 & 0.0430 & 0.0301 & 0.0010 & 0.9986 & 1.0000 \\
\hline
\end{tabular}

Table 8. The K-S test results and CDF values of sample 2\#

\begin{tabular}{|c|c|c|c|c|c|c|c|}
\hline \multirow{2}{*}{ Size } & \multicolumn{2}{|c|}{ Truncated interval } & \multirow{2}{*}{$D_{n, 0.05}$} & \multicolumn{2}{c|}{ K-S test results } & \multicolumn{2}{c|}{ CDF values } \\
\cline { 2 - 5 } & Left & Right & & Weibull & NID & Weibull & NID \\
\hline 15 & 0.4664 & 12.5077 & 0.3380 & 0.3336 & 0.0730 & 1.0000 & 1.0000 \\
\hline 20 & 1.7339 & 12.8139 & 0.2940 & 0.1857 & 0.0500 & 0.9995 & 1.0000 \\
\hline 30 & 1.5277 & 12.2911 & 0.2420 & 0.1865 & 0.0344 & 0.9997 & 1.0000 \\
\hline 50 & 3.3735 & 11.1177 & 0.1923 & 0.1238 & 0.0200 & 0.9828 & 1.0000 \\
\hline 100 & 1.9711 & 11.8769 & 0.1360 & 0.0552 & 0.0137 & 0.9988 & 1.0000 \\
\hline 200 & 2.2851 & 11.8036 & 0.0962 & 0.0310 & 0.0050 & 0.9976 & 1.0000 \\
\hline 500 & 1.9713 & 11.8592 & 0.0608 & 0.0475 & 0.0020 & 0.9988 & 1.0000 \\
\hline 1000 & 2.2985 & 11.9048 & 0.0430 & 0.0209 & 0.0010 & 0.9976 & 1.0000 \\
\hline
\end{tabular}




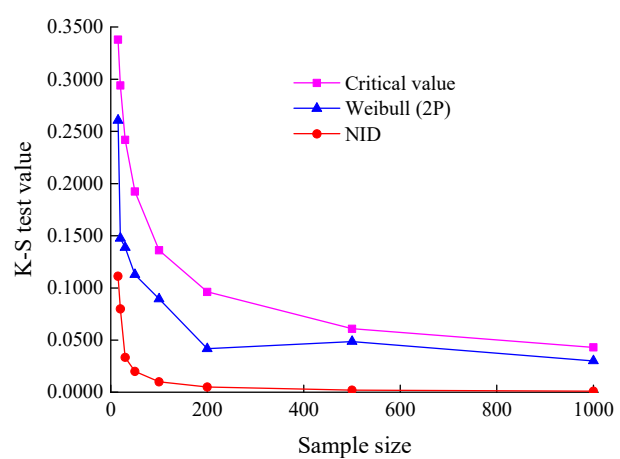

a) Sample $1 \#$

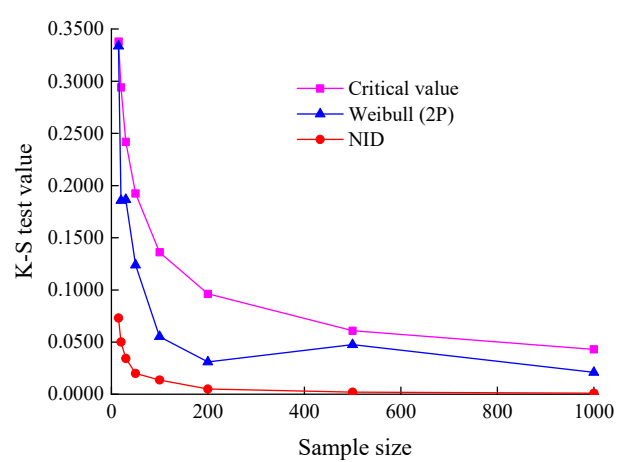

b) Sample 2\#

Fig. 4. K-S test results of the simulated data with the sample size

Table 9. The results of the chi-square tests of samples 1\# and 2\#

\begin{tabular}{|c|c|c|c|c|c|c|}
\hline \multirow{2}{*}{$\begin{array}{c}\text { The sizes of MC } \\
\text { data }\end{array}$} & \multirow{2}{*}{$\begin{array}{c}\text { The number of } \\
\text { intervals }\end{array}$} & $\begin{array}{c}\text { Critical value for } \\
\text { Weibull }\end{array}$ & Weibull & $\begin{array}{c}\text { Critical value for } \\
\text { NID }\end{array}$ & NID \\
\hline \multirow{4}{*}{$1 \#$} & 50 & 7 & 9.4877 & 7.5426 & 12.5916 & 0.5208 \\
\cline { 2 - 7 } & 100 & 10 & 14.0671 & 8.8863 & 16.9190 & 1.2689 \\
\cline { 2 - 7 } & 200 & 14 & 19.6751 & 11.1422 & 22.3621 & 0.1825 \\
\cline { 2 - 7 } & 500 & 22 & 30.1435 & 15.0571 & 32.6705 & 0.5096 \\
\cline { 2 - 7 } & 1000 & 31 & 41.3372 & 30.2494 & 43.7729 & 0.6558 \\
\hline \multirow{4}{*}{$2 \#$} & 50 & 7 & 9.4877 & 4.7083 & 12.5916 & 1.0037 \\
\cline { 2 - 7 } & 100 & 10 & 14.0671 & 4.0272 & 16.9190 & 0.6340 \\
\cline { 2 - 7 } & 200 & 14 & 19.6751 & 5.1070 & 22.3621 & 0.4919 \\
\cline { 2 - 7 } & 500 & 22 & 30.1435 & 9.6412 & 32.6705 & 0.3132 \\
\cline { 2 - 7 } & 1000 & 31 & 41.3372 & 28.9021 & 43.7729 & 0.5684 \\
\hline
\end{tabular}

The change in the chi-square test results with an increase in the sample size are shown in Fig. 5 for the simulated samples. It can be seen that both the NID distribution and Weibull distribution have passed the chi-square test. However, the test results of the NID distribution are considerably lower than those of the Weibull distribution; the test results of the Weibull distribution are one to two orders of magnitude greater than those of the NID distribution. Thus, the goodness of fit of the NID distribution is superior to that of the Weibull distribution. Moreover, the test results of the NID distribution are much more stable than those of the Weibull distribution.

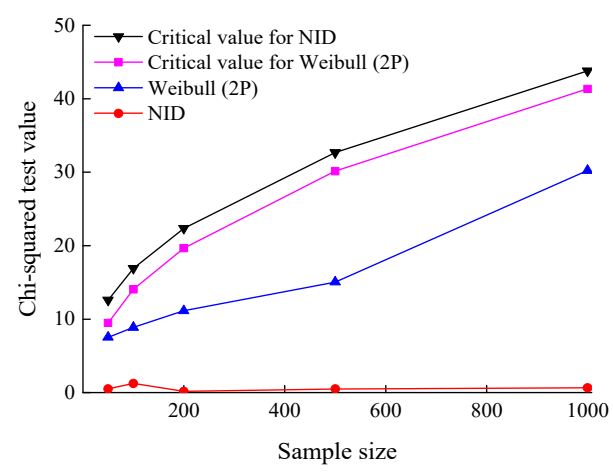

a) Sample 1\#

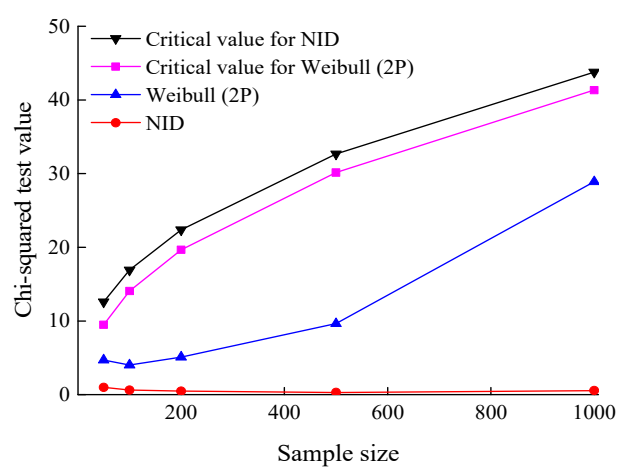

b) Sample 2\#

Fig. 5. The chi-square test results of the simulated data with the sample size

The CDF curves of the NID distribution and Weibull distribution for the simulated data of sample 1\# are shown in Fig. 6. It is easy to see that, with an increase in the sample size, the CDF 
curves of the NID distribution are always closer to the empirical cumulative distribution function (EDF) curves than those of the Weibull distribution. Clearly, when the sample size is equal to 1000 , the curves of the NID, Weibull and empirical distributions are nearly coincident.

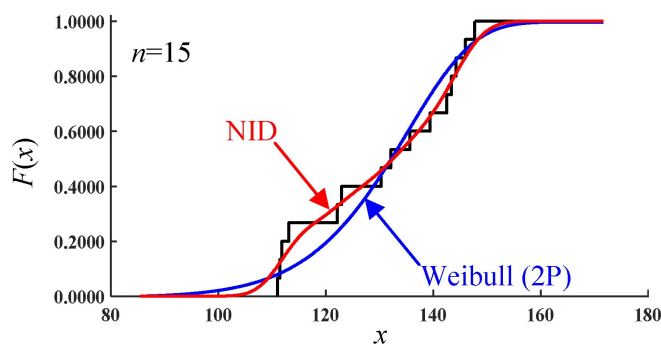

a)

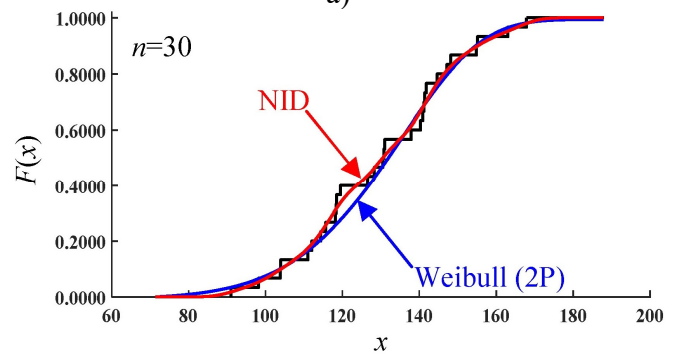

c)

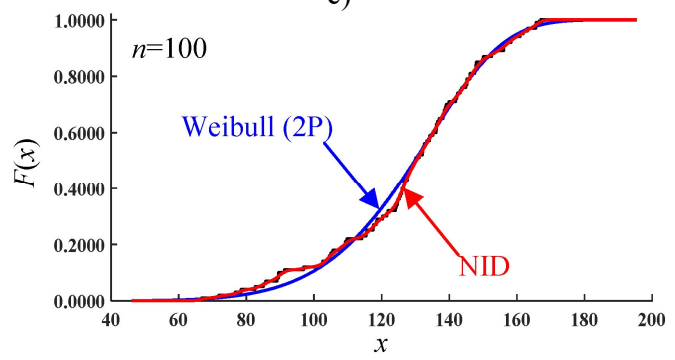

e)

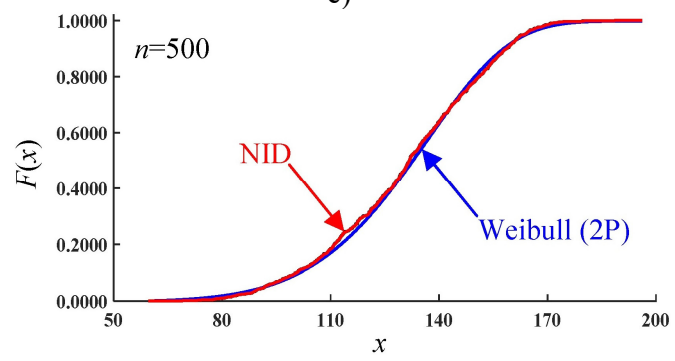

g)

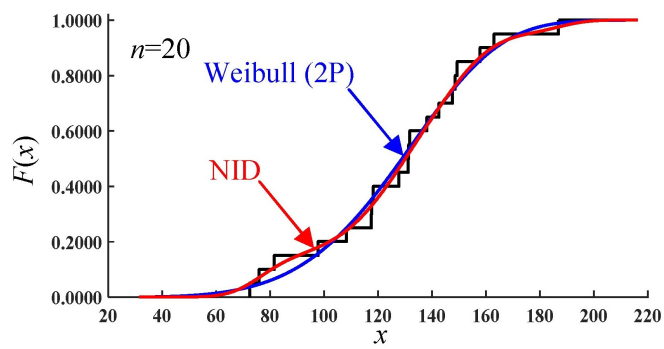

b)

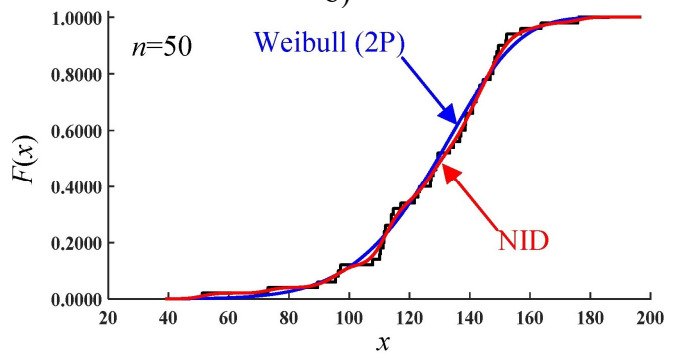

d)

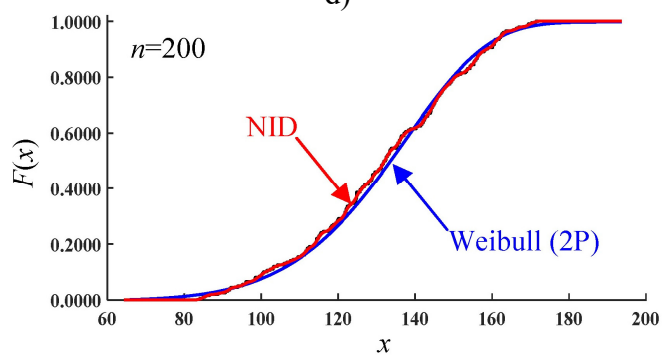

f)

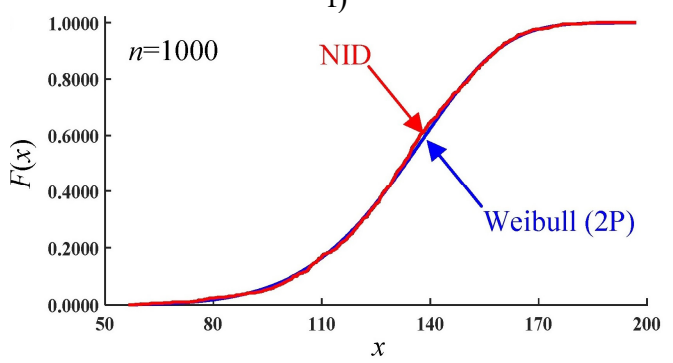

h)

Fig. 6. Comparative CDF curves of the simulated data of sample 1\# (sample size $n$ )

The CDF values for simulated samples $1 \#$ and $2 \#$ with different sizes are shown in Tables 7 and 8 , and the trends of the CDF values with sample size are shown in Fig. 7. Clearly, with an increase in the sample size, the cumulative probability values of the NID distribution are always equal to 1.0000 and are completely unaffected by the sample size. However, the cumulative probability values of the Weibull distribution are generally less than 1.0000 , and there is a considerable amount of volatility when the sample size increases. It is evident from the above analysis that the NID distribution has a higher fitting precision and wider applicability. 
INFERENCE OF THE OPTIMAL PROBABILITY DISTRIBUTION MODEL FOR GEOTECHNICAL PARAMETERS BY USING WEIBULL AND NID DISTRIBUTIONS. FENGQIANG GONG, TIANCHENG WANG, SHANYONG WANG

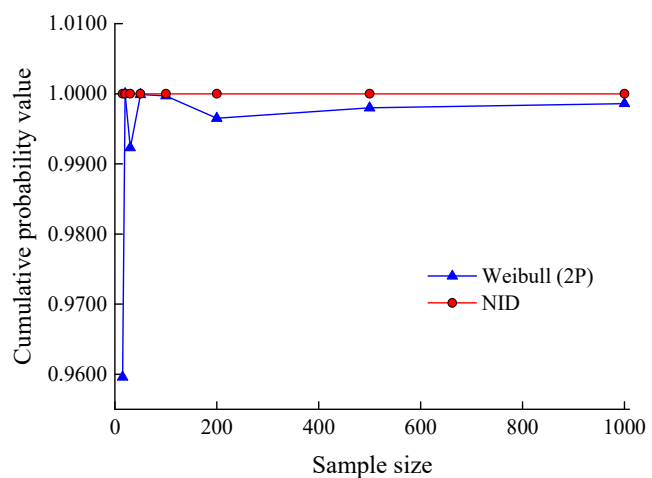

a) Sample $1 \#$

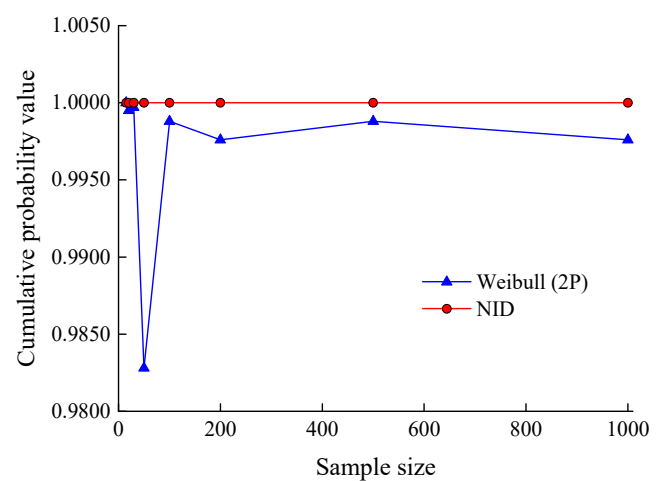

b) Sample 2\#

Fig. 7. Cumulative probability values of the simulated data with the sample size

\section{Discussion}

In the truncated interval, the cumulative probability values of classical distributions are usually less than 1.0000. To solve this problem, the normalization of the truncated classical distribution was introduced. The basic principle of normalized distribution is introduced as follows:

$$
\begin{aligned}
& \tilde{f}(x)=k f(x), \quad L<x<R, \\
& k=\frac{1}{F(R)-F(L)},
\end{aligned}
$$

where $\tilde{f}(x)$ is the normalized PDF, $F(x)$ is the cumulative PDF, $f(x)$ is the classical PDF, $x$ is the value of the sample, and $R$ and $L$ are the maximum and minimum values of the sample, respectively.

Table 10. The results of K-S test values of the normalized Weibull distributions

\begin{tabular}{|c|c|c|c|c|c|c|}
\hline \multicolumn{2}{|c|}{ Sample } & $D_{n, 0.05}$ & $k$ & Normalized Weibull & NID & Weibull \\
\hline \multirow{3}{*}{ Actual } & $1 \#$ & 0.3100 & 1.0047 & 0.1046 & 0.0683 & 0.0969 \\
\cline { 2 - 7 } & $2 \#$ & 0.2420 & 1.0030 & 0.1475 & 0.0576 & 0.1495 \\
\hline \multirow{5}{*}{ MC 1\# } & 15 & 0.3380 & 1.0038 & 0.1670 & 0.1113 & 0.2607 \\
\cline { 2 - 8 } & 20 & 0.2940 & 1.0006 & 0.1031 & 0.0799 & 0.1476 \\
\cline { 2 - 8 } & 30 & 0.2420 & 1.0065 & 0.1225 & 0.0334 & 0.1388 \\
\cline { 2 - 8 } & 50 & 0.1923 & 1.0002 & 0.0736 & 0.0200 & 0.1127 \\
\cline { 2 - 8 } & 100 & 0.1360 & 1.0005 & 0.0769 & 0.0100 & 0.0894 \\
\cline { 2 - 8 } & 200 & 0.0962 & 1.0031 & 0.0428 & 0.0050 & 0.0417 \\
\cline { 2 - 7 } & 500 & 0.0608 & 1.0027 & 0.0334 & 0.0020 & 0.0487 \\
\cline { 2 - 7 } & 1000 & 0.0430 & 1.0018 & 0.0287 & 0.0010 & 0.0301 \\
\cline { 2 - 7 } & 15 & 0.3380 & 1.0002 & 0.1579 & 0.0730 & 0.3336 \\
\cline { 2 - 7 } & 20 & 0.2940 & 1.0008 & 0.1409 & 0.0500 & 0.1857 \\
\cline { 2 - 7 } & 30 & 0.2420 & 1.0001 & 0.1091 & 0.0344 & 0.1865 \\
\cline { 2 - 7 } & 50 & 0.1923 & 1.0051 & 0.0722 & 0.0200 & 0.1238 \\
\cline { 2 - 7 } & 100 & 0.1360 & 1.0008 & 0.0494 & 0.0137 & 0.0552 \\
\cline { 2 - 7 } & 200 & 0.0962 & 1.0014 & 0.0432 & 0.0050 & 0.0310 \\
\cline { 2 - 7 } & 500 & 0.0608 & 1.0011 & 0.0292 & 0.0020 & 0.0475 \\
\cline { 2 - 7 } & 1000 & 0.0430 & 1.0020 & 0.0207 & 0.0010 & 0.0209 \\
\hline
\end{tabular}

The K-S test values of the normalized Weibull distribution for a $95 \%$ confidence level are shown in Table 10. The sequence of the K-S test value of actual sample 1\# is 0.0683 (NID) $<0.0969$ (Weibull) < 0.1046 (normalized Weibull) $<0.3100$ (Critical value). The sequence of the 
K-S test value of actual sample $2 \#$ is 0.0576 (NID) $<0.1475$ (normalized Weibull) $<0.1495$ (Weibull) $<0.2420$ (Critical value). It can be found that all of the K-S test values pass the testing. However, all of the K-S test values of the normalized Weibull distribution are much more than those of the NID distribution, which indicates that the fitting ability of NID distribution is better than that of normalized Weibull distribution.

\section{Conclusions}

To accurately approximate the PDFs for geotechnical parameters, the NID method was introduced; several conclusions of this study are given below.

1) The PDFs of two sets of geotechnical samples were fitted with the NID distribution and Weibull distribution. The results show that, for the K-S test results, the chi-square test results and the cumulative probability values, the NID distribution is more accurate than the Weibull distribution. In addition, compared with the PDF curves of the Weibull distribution, those of the NID distribution can overcome the single-peak feature of the classical distributions and agree more closely with those of the actual distribution.

2) The effect of the sample size on the fitting accuracy for the NID distribution and Weibull distribution was investigated with the MC method, and eight simulated samples were produced. It can be found that with an increase in the sample size, the K-S test results of the NID distribution are all lower than those of the Weibull distribution. In addition, its convergence speed and stability are superior to those of the Weibull distribution. The cumulative probability values of the NID distribution are always equal to 1.0000 in the truncated interval and are unaffected by the sample size. However, the cumulative probability values of the Weibull distribution are generally less than 1.0000 and are unstable.

3) The comparison of the fitting accuracy between the NID distribution and the normalized Weibull distribution was also discussed, and the results show that, even if the cumulative probability values are equal to 1 for those two distributions, the fitting accuracy of the NID distribution is still higher than that of normalized Weibull distribution.

\section{Acknowledgements}

This research was financially supported by the Natural Science Foundation of China (Grant No. 41102170) and the Fundamental Research Funds for the Central Universities of Central South University (Grant No. 2017zzts536).

\section{References}

[1] Whitman R. V. Organizing and evaluating uncertainty in geotechnical engineering. Journal of Geotechnical and Geoenvironmental Engineering, Vol. 126, Issue 7, 2000, p. 583-593.

[2] Christian J. T., Baecher G. B. Unresolved problems in geotechnical risk and reliability. Georisk, Vol. 418, 2011, p. 50-63.

[3] Christian J. T. Geotechnical engineering reliability: how well do we know what we are doing? Journal of Geotechnical and Geoenvironmental Engineering, Vol. 130, Issue 10, 2004, p. 985-1003.

[4] Baecher G. B., Christian J. T. Reliability and Statistics in Geotechnical Engineering. John Wiley and Sons, 2005.

[5] Phoon K. K., Ching J. Y. Risk and Reliability in Geotechnical Engineering. CRC Press, 2014.

[6] Christian J. T., Urzua A. Probabilistic evaluation of earthquake induced slope failure. Journal of Geotechnical and Geoenvironmental Engineering, Vol. 124, Issue 11, 1998, p. 1140-1143.

[7] Al-Homoud A. S., Tahtamoni W. W. Reliability analysis of three-dimensional dynamic slope stability and earthquake-induced permanent displacement. Soil Dynamics and Earthquake Engineering, Vol. 19, Issue 2, 2000, p. 91-114.

[8] Zhang L., Lin C. M., Xu C., et al. A method for calculating reliability of soil slope stability under dynamic loading. Journal of Disaster Prevention and Mitigation Engineering, Vol. 25, Issue 2, 2005, p. 178-181, (in Chinese). 
[9] Li Y., Gong W. H., Chen X. L., et al. Dynamic reliability analysis on rock slopes under earthquake. Journal of Civil Engineering and Management, Vol. 33, Issue 3, 2016, p. 94-98, (in Chinese).

[10] Huang Y., Xiong M. Dynamic reliability analysis of slopes based on the probability density evolution method. Soil Dynamics and Earthquake Engineering, Vol. 94, 2017, p. 1-6.

[11] Xiong M., Huang Y. Stochastic seismic response and dynamic reliability analysis of slopes: a review. Soil Dynamics and Earthquake Engineering, Vol. 100, 2017, p. 458-464.

[12] Wang Y., Cao Z. J., Au S. K. Efficient Monte Carlo simulation of parameter sensitivity in probabilistic slope stability analysis. Computers and Geotechnics, Vol. 37, Issue 7, 2010, p. 1015-1022.

[13] Lü Q., Low B. K. Probabilistic analysis of underground rock excavations using response surface method and SORM. Computers and Geotechnics, Vol. 38, Issue 8, 2011, p. 1008-1021.

[14] Su Y. H., Fang Y., Li S., et al. A one-dimensional integral approach to calculating the failure probability of geotechnical engineering structures. Computers and Geotechnics, Vol. 90, 2017, p. 85-95.

[15] Deng J., Li X. B., Gu G. S. A distribution-free method using maximum entropy and moments for estimating probability curves of rock variables. International Journal of Rock Mechanics and Mining Sciences, Vol. 41, Issue 3, 2004, p. 127-132.

[16] Li X. B., Gong F. Q. A method for fitting probability distributions to engineering properties of rock masses using Legendre orthogonal polynomials. Structural Safety, Vol. 31, Issue 4, 2009, p. 335-343.

[17] Wu X. Z. Trivariate analysis of soil ranking-correlated characteristics and its application to probabilistic stability assessments in geotechnical engineering problems. Soils and Foundations, Vol. 53, Issue 4, 2013, p. 540-556.

[18] Do M. Comparative analysis on mean life reliability with functionally classified pavement sections. KSCE Journal of Civil Engineering, Vol. 15, Issue 2, 2011, p. 261-270.

[19] Dianty M. A., Yahaya A. S., Ahmad F. Probability distribution of engineering properties of soil at telecomunication sites in Indonesia. International Journal of Scientific Research in Knowledge, Vol. 2, Issue 3, 2014, p. 143-150.

[20] Ardeleanu L. F. Statistical models of the seismicity of the Vrancea region, Romania. Natural Hazards, Vol. 19, Issues 2-3, 1999, p. 151-164.

[21] Huang C. Principle of information diffusion. Fuzzy Sets and Systems, Vol. 91, Issue 1, 1997, p. 69-90.

[22] Huang C. F., Shi Y. Towards Efficient Fuzzy Information Processing. Springer-Verlag Berlin Heidelberg GmbH, 2002.

[23] Li Q., Zhou J. Z., Liu D. H., et al. Research on flood risk analysis and evaluation method based on variable fuzzy sets and information diffusion. Safety Science, Vol. 50, Issue 5, 2012, p. 1275-1283.

[24] Xu L. F., Xu X. G., Meng X. W. Risk assessment of soil erosion in different rainfall scenarios by RUSLE model coupled with information diffusion model: a case study of Bohai Rim, China. Catena, Vol. 100, 2013, p. 74-82.

[25] Li Q., Zhou J. Z., Liu D. H., et al. Disaster risk assessment based on variable fuzzy sets and improved information diffusion method. Human and Ecological Risk Assessment an International Journal, Vol. 19, Issue 4, 2013, p. 857-872.

[26] Jiang L. C., Du W. W. Distribution characteristics of rock mass strength subjected to acid attack. Journal of Kunming University of Science and Technology, Vol. 35, Issue 4, 2010, p. 6-10, (in Chinese).

[27] Xu R. P., Fu H. M., Gao Z. T. Estimation of parameters and confidence limits for wind speed probability distribution. Journal of Applied Statistics and Management, Vol. 14, Issue 3, 1995, p. 32-35, (in Chinese).

[28] Ang A.-H. S., Tang W. H. Probability Concepts in Engineering: Emphasis on Applications to Civil and Environmental Engineering. Second Edition, John Wiley and Sons, 2007.

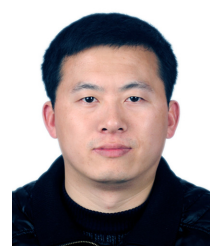

Fengqiang Gong received Ph.D. degree in mining engineering from Central South University, P. R. China, in 2010. Now he works at Central South University as an Associate Professor. His current research interests include reliability of geotechnical engineering and rock mechanics. 
Tiancheng Wang received bachelor's degree in mining engineering from Henan Polytechnic University, P. R. China, in 2016. Now he is a master's degree candidate of Central South University. His current research interest is reliability of geotechnical engineering.

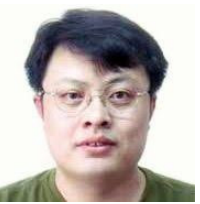

Shanyong Wang received Ph.D. degree in geotechnical engineering from City University of Hong Kong, Hong Kong, in 2007. Now he works at the University of Newcastle as an Associate Professor. His current research interests include stability analysis of geotechnical engineering, rock fracturing, compaction/fracturing grouting and soil nail. 